\title{
Protein Splicing Abnormality
}

National Cancer Institute

\section{Source}

National Cancer Institute. Protein Splicing Abnormality. NCI Thesaurus. Code C45595.

A change in the amino acid sequence of a wild-type protein, resulting from aberrant excision of in-frame protein sequences (inteins) from a precursor protein followed by ligation of the flanking sequences (exteins). 